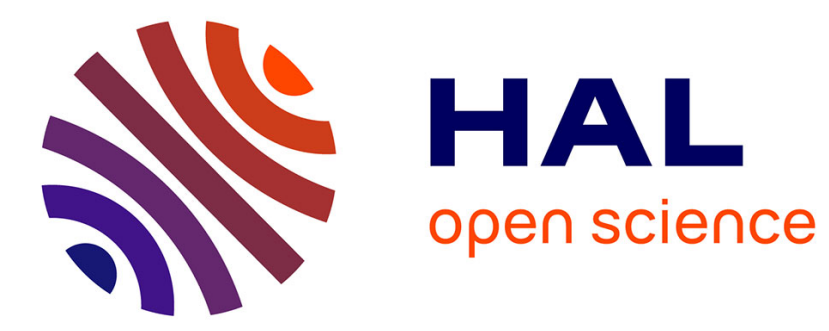

\title{
Seismic Metashielding by a Line of Resonators Over a Granular Layer
}

\author{
Pierric Mora, Mathieu Chekroun, Vincent Tournat
}

\section{To cite this version:}

Pierric Mora, Mathieu Chekroun, Vincent Tournat. Seismic Metashielding by a Line of Resonators Over a Granular Layer. Physical Review Applied, 2021, 16 (4), 9 p. 10.1103/physrevapplied.16.044002 . hal-03382305

\section{HAL Id: hal-03382305 https://hal.science/hal-03382305}

Submitted on 18 Oct 2021

HAL is a multi-disciplinary open access archive for the deposit and dissemination of scientific research documents, whether they are published or not. The documents may come from teaching and research institutions in France or abroad, or from public or private research centers.
L'archive ouverte pluridisciplinaire HAL, est destinée au dépôt et à la diffusion de documents scientifiques de niveau recherche, publiés ou non, émanant des établissements d'enseignement et de recherche français ou étrangers, des laboratoires publics ou privés. 


\title{
Seismic Metashielding by a Line of Resonators Over a Granular Layer
}

\author{
Pierric Mora $\odot,{ }^{*}$ Mathieu Chekroun $\odot$, and Vincent Tournat๑ \\ Laboratoire d'Acoustique de l'Université du Mans (LAUM), UMR 6613, Institut d'Acoustique -Graduate School \\ (IA-GS), CNRS, Le Mans Université, France
}

(Received 22 July 2021; revised 14 September 2021; accepted 16 September 2021; published 4 September 2021)

\begin{abstract}
Seismic metamaterials have been the subject of much recent interest, with notable conceptual advances and the demonstration of possible full-scale effectiveness in earthquake protection. Here, through a fine analysis of the dispersion properties and behavior of resonators introduced in a laboratory scale setup, we propose a strategy for the design of an efficient metaseismic barrier. When positioned onto an unconsolidated granular layer, rigid masses of nontrivial shape become elastic resonators by coupling with the relatively soft propagation medium. Additionally, they affect the mechanical boundary conditions and subsurface medium properties through the added weight. We demonstrate that all these ingredients can be rationally combined to improve the shielding effect and control the seismic waves.
\end{abstract}

DOI: 10.1103/PhysRevApplied.16.044002

\section{INTRODUCTION}

Seismic metamaterials have emerged in the last decade as strategies to control elastic waves propagating close to the surface of large-scale mechanical systems [1]. Similar to metamaterials in various wave systems, the use of local resonators, achieving noticeable effects on traveling waves even with engineered structures of subwavelength dimensions, is particularly appealing for seismic waves or even soil vibrations, which deal with typical wavelengths ranging from meters to kilometers [2]. In this context, various types of resonator arrangements have been proposed to attenuate or convert waves propagating in elastic layers, either at large scale [2], including forests [3,4], for experiments at the lab scale [5-7], or using theoretical and numerical approaches [8-13].

Of particular relevance for laboratory-scale experiments on seismic metamaterials is the implementation of unconsolidated granular layers, which add complexity in the elastic propagation layer [14-18]. Indeed, due to the contact nonlinearity between grains and the hydrostatic pressure increase with depth, an elastic property gradient forms in unconsolidated granular layers [19-21]. As a result, the wave-propagation velocities increase with depth.

Such inhomogeneity of the elastic layer, whose characteristics are now reasonably known in the form of an indepth power law for both the longitudinal and shear-wave velocities, leads to the propagation of guided modes called guided surface acoustic modes (GSAMs) or RayleighHertz modes [14,22]. These modes have been extensively studied in laboratory configurations with granular models composed of spherical glass beads of size approximately

\footnotetext{
*pierric.mora@univ-eiffel.fr
}

$100 \mu \mathrm{m}$, offering a good fit between theory, simulations, and experiments [5,14-18]. The interest to study seismic metamaterials with granular layers in the laboratory comes from the existence of these GSAM modes. Indeed, in a real geological environment, the inhomogeneities, stratifications, also give rise to the existence of guided modes, to in-depth refraction, in other words, to a complexity that is not present in a homogeneous elastic half-space, supporting only the Rayleigh wave together with bulk modes. Thus, taking into account an elastic inhomogeneity requires the design of advanced seismic metamaterials, capable of operating on several propagation modes, for example, to create a metaseismic barrier.

In this paper, we study experimentally and numerically a metaseismic barrier composed of a line of elastic resonators of simple design, but operating on the two main guided modes polarized in the sagittal plane of an inhomogeneous granular layer. These resonators are rigid masses of nontrivial shape added to the granular surface, which become elastic resonators by coupling with the relatively soft propagation medium. We show, through comparisons between experiments and numerical simulations, and based on elements of theory, that the processes leading to the hybridization of the first two GSAMs are due to the joint effects of the hardening of the medium under the resonators (effect of the added stress on a column of nonlinear granular media) and of two distinct resonances, dependent on the geometry of the mass, which induce a Neumann to Dirichlet transition for the boundary condition on both components of the dynamic surface displacement. Finally, we demonstrate experimentally a significant attenuation effect of the first two modes of propagation, achieved by a single line of resonators and propose possible extensions of this work. 


\section{INITIAL CONCEPT AND WAVE PROPAGATION WITHOUT THE METABARRIER}

\section{A. Initial concept}

The idea of the design presented here is that when a massive body is deposited at the surface of the half-space, the granular medium is slightly compressed and turns into a soft elastic solid below the contact area with the mass. The expected behavior is then that of a mass-spring-dashpot system - the role of the spring being played by the granular medium itself and the attenuation being due to the radiation of waves into the half-space. Several resonances can be expected in relation to the different inertial characteristics of the body (mass and angular momenta) and interface stiffnesses (normal, shear, torsion). Among those, two are of particular relevance since they involve free components of the boundary condition and are hence prone to hybridize the propagation of guided waves: a piston mode in which the mass moves vertically, and a shear mode in which the contact area is mostly polarized in the plane of the surface. If properly tuned, the parameters defining the added solid object can lead to a simple resonant system able to affect the boundary conditions seen by all wave components simultaneously. Although approximate analytical expressions of contact resonances are known for homogeneous half-spaces (see Refs. [23,24] for reviews and Refs. $[25,26]$ for examples of applications to metasurfaces), our case is significantly more complex due to the strong heterogeneities in the stratum and their interplay with the mass, so that this work of parameter tuning is entirely done numerically. Our experimental setup is presented in Fig. 1 and described in details in Appendix A.

\section{B. Wave propagation in the granular layer without scatterers}

At large wavelengths compared to the size of the beads, a granular medium may be described as an elastic medium whose stiffness depends on the applied pressure as a power law (see Refs. [14,19,20,22]). Due to the increase of the hydrostatic pressure with depth, a granular halfspace is then described with a strong gradient of bulk-wave velocities:

$$
c_{P, S}(z)=\gamma_{P, S}(\rho g z)^{\alpha_{P, S}},
$$

in which $c_{P}\left(c_{S}\right)$ is the longitudinal (shear) wave velocity, $z$ the depth coordinate ( $z=0$ is a stress-free interface), $g=9.81 \mathrm{~m} / \mathrm{s}^{2}$ the standard gravity, and $\alpha_{P, S}$ and $\gamma_{P, S}$ are characteristic constants of the power law. In this paper we take $\alpha_{P}=\alpha_{S}=\alpha=0.31, \gamma_{P}=14.4$, and $\gamma_{S}=6.4$ in accordance with previous experimental works (see Refs. [5,14-18]). This slow-to-fast gradient has a guiding effect. In contrast to other heterogeneous half-spaces supporting surface modes, this power-law medium has the peculiar feature not to possess any characteristic length scale. As a consequence, no cutoff frequency exist and an infinite number of channels of surface waves are open at any given frequency. Furthermore, the eigenfrequencies scale with wave number as $[19,20]$

$$
\frac{f_{n, 2}}{f_{n, 1}}=\left(\frac{k_{2}}{k_{1}}\right)^{1-\alpha},
$$

in which $n$ is the modal order, so that it is enough to obtain them at one wave number and then post-treat the results using Eq. (2) to obtain the eigenfrequencies and mode shapes at other wave numbers, or conversely to obtain the eigen-wave-numbers and mode shapes as a function of the frequency. The first two fundamental modes $n=1$ and $n=2$ are of particular interest in this work, as the energy they carry is most strongly localized at the surface. Higher-order modes propagate faster and with a deeper localization. Figure 2 depicts typical experimental signals, as well as theoretical dispersion relations and mode shapes for the first three modes - obtained with a finite-element software.

\section{NUMERICAL MODELING OF THE METABARRIER}

\section{A. Model of the consolidation caused by a massive object}

The basic assumption we make to model this interaction is that the granular medium consolidates only within a cylindrical region below the object whose cross section is constant and equal to the contact area. The magnitude of this consolidation is assumed to follow the constitutive law given in Eq. (1). In this consolidated region, the bulk velocities express as

$$
c_{P, S}^{\mathrm{cons}}=\gamma_{P, S}\left[\rho g\left(z+z_{0}\right)\right]^{\alpha_{P, S}}
$$

with the consolidation depth

$$
z_{0}=\frac{M}{\rho S},
$$

where $M$ is the mass of the solid and $S$ the contact area with the granular medium. Away from this region, the bulk velocities are kept unchanged and are given by Eq. (1). Of course, this is a coarse description of what really happens as one expects the actual response of the substrate to be rather conical. However, we find out via parametric studies that the shape of the consolidation is a parameter of minor influence relatively to others, so that we decide to rely on this simplistic description. In addition, there is no current knowledge about the shape and characteristics of this affected area and how this depends on the preparation procedure. 

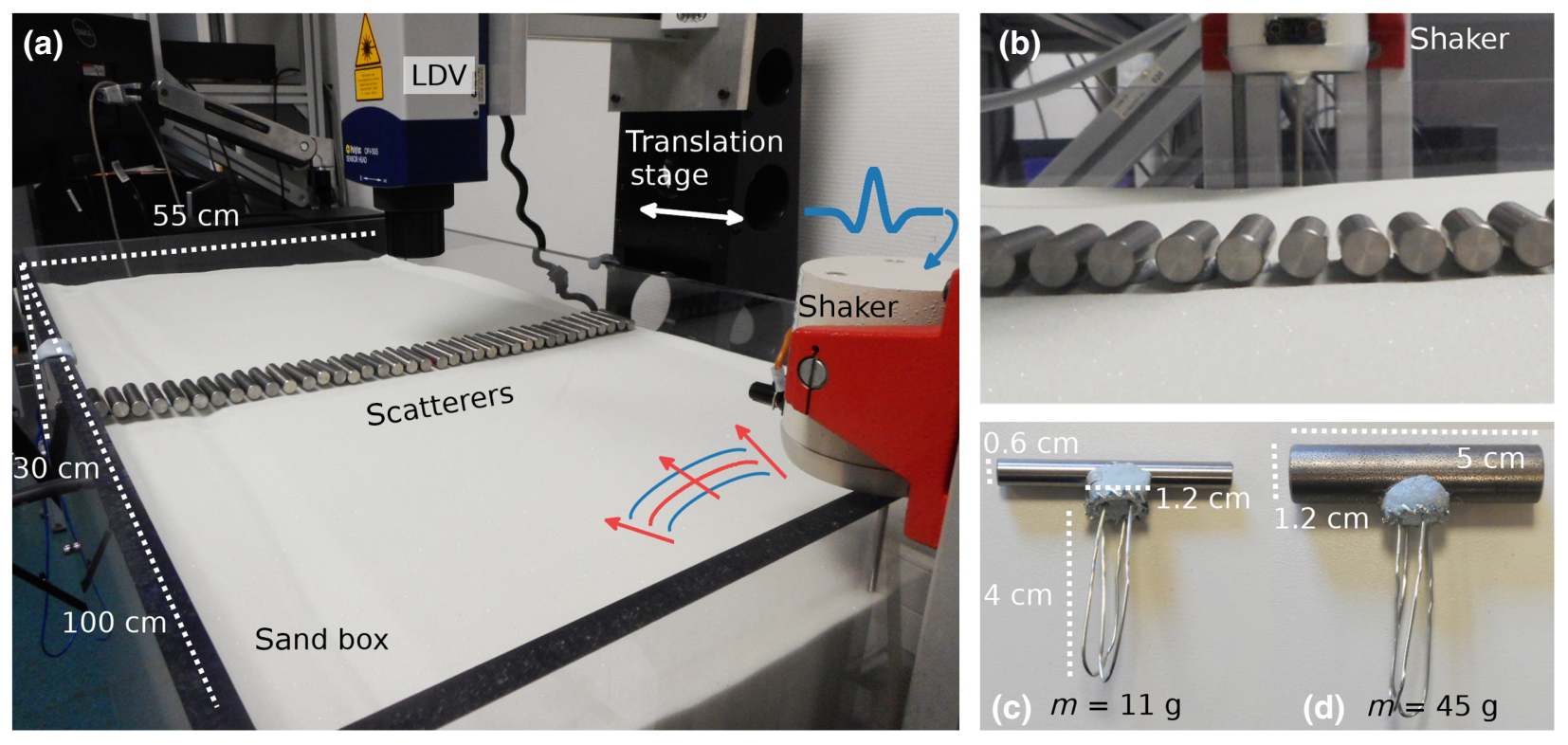

FIG. 1. Experimental setup showing one line of heavy scatterers [(a),(b)]. A light (c) and a heavy (d) scatterer.

\section{B. Dispersion analysis for a periodic arrangement of scatterers}

The numerical results presented below are obtained from a two-dimensional (2D) finite-element (FE) model, after transposition and validation from a three-dimensional (3D) model. The geometrical dimensions of the 3D model directly represent the scatterers used in the experiments, with the assumption that the spacing between neighboring scatterers is (almost) zero. Corresponding rules are used to define the 2D model, which we now describe. A schematic representation is shown in Fig. 3(a). All FE models are built using COMSOL Multiphysics ${ }^{\circledR}$, with the Structural Mechanics module.

The 2D model consists in a unit cell of size $l_{\mathrm{UC}} \times h_{\mathrm{UC}}=$ $1.2 \times 70 \mathrm{~cm}^{2}$ filled with an elastic medium of mass density $\rho=1560 \mathrm{~kg} / \mathrm{m}^{3}$ and space-dependent elastic constants deduced from Eq. (3) on a column below the contact with the added solid, and from Eq. (1) elsewhere. The
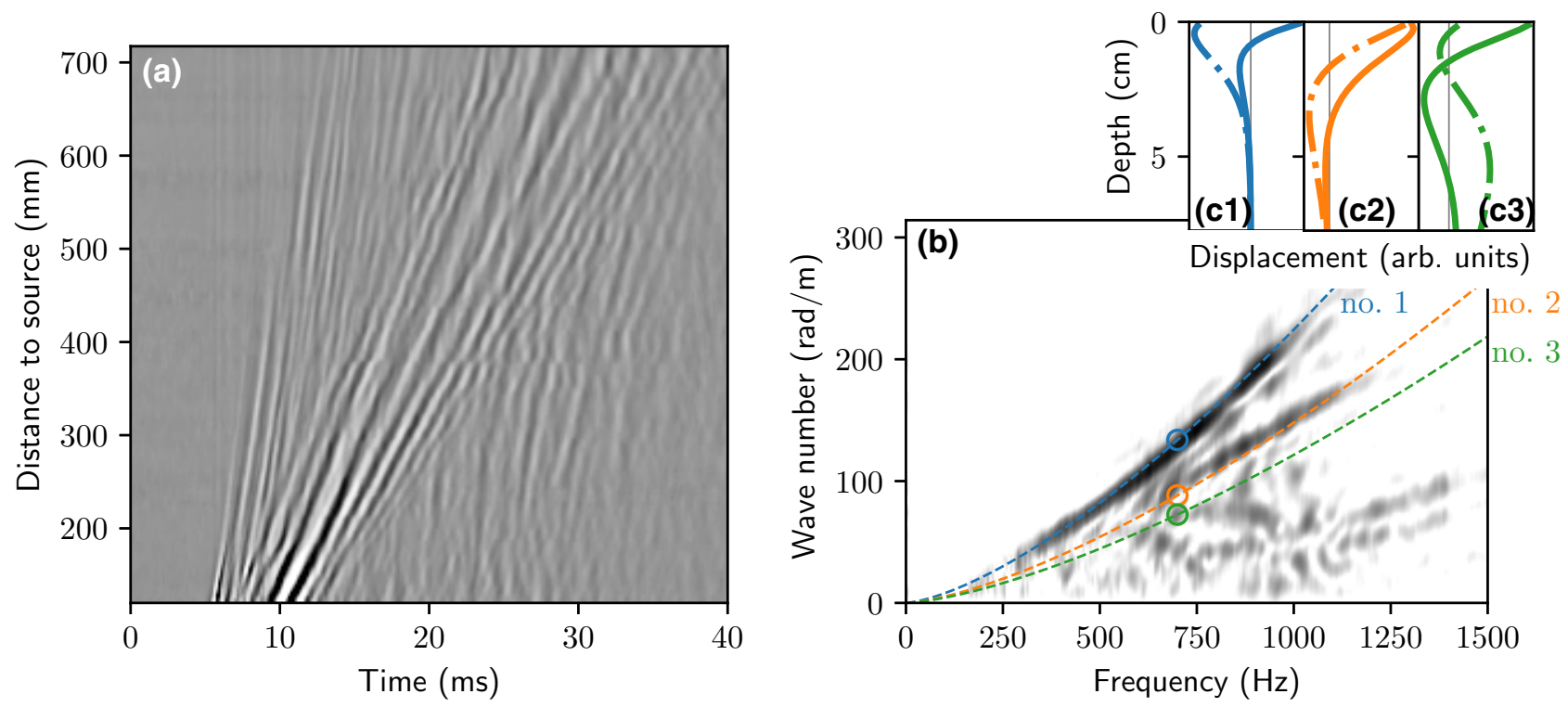

FIG. 2. Vertical velocity at the surface of the granular medium generated by a Ricker impulse, in the absence of scatterers. (a) Space-time signals, (b) experimental and the first three theoretical dispersion curves, (c1), (c2), and (c3) corresponding theoretical mode shapes at $700 \mathrm{~Hz}$ : (solid) longitudinal $v_{x}$ and (dashed) vertical $v_{z} / i$ components. $\lambda_{1}(700 \mathrm{~Hz})=4.7 \mathrm{~cm}, \lambda_{2}(700 \mathrm{~Hz})=7.1 \mathrm{~cm}$, and $\lambda_{3}(700 \mathrm{~Hz})=8.7 \mathrm{~cm}$. 
(a)

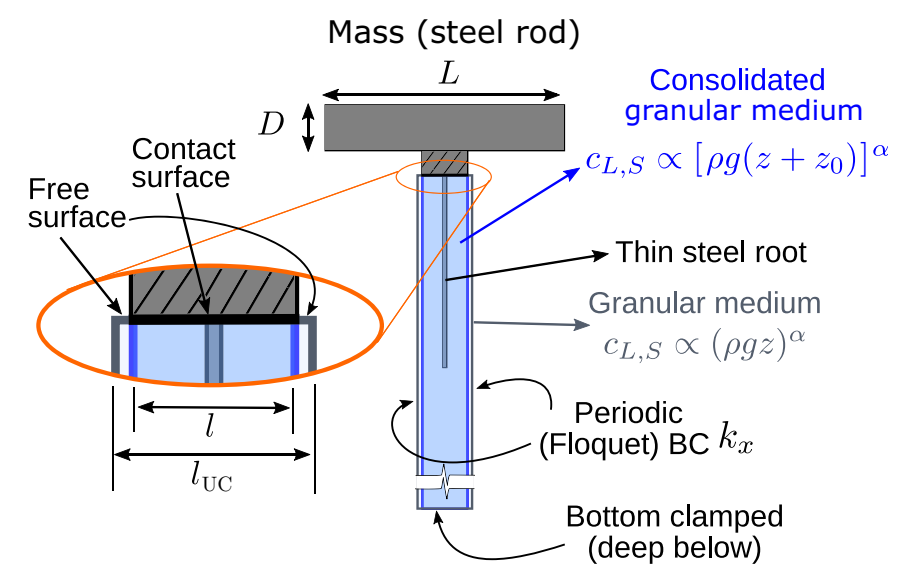

(b)

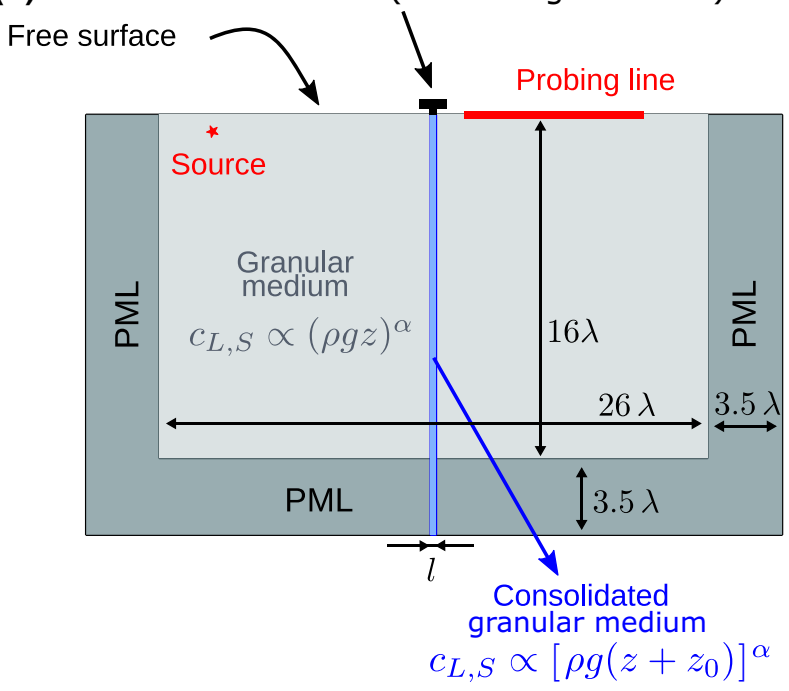

FIG. 3. Scheme of the FE modeling. (a) Unit cell for 2D dispersion analysis. (b) $2 \mathrm{D}$ configuration for the reflection and transmission analysis through one resonator line. The dimension parameter $\lambda$ refers to the wavelength of the lowest-order mode $n=1$ at the operating frequency. BC, boundary conditions.

mesh is refined near the surface to follow the strong variations of properties - element sides are there required to be no larger than $4 \%$ of the shortest wavelength, giving a total of roughly $2 \times 10^{4}$ degrees of freedom. Floquet periodic boundary conditions are applied on lateral boundaries, while the (fictitious) bottom end is clamped and the top interface is stress-free. The scatterer is an elastic solid of rectangular shape of size $L \times D^{(2 \mathrm{D})}$, with a length $L=5 \mathrm{~cm}$ and a height $D^{(2 \mathrm{D})}$ that we specify further, and with mechanical constants that are typical of steel (density of $7800 \mathrm{~kg} / \mathrm{m}^{3}$, Young's modulus of $200 \mathrm{GPa}$, Poisson's ratio of 0.3 ). From these latter constants only the density really matters as the frequency range lies far below the first elastic resonances of the object, which indeed behaves as a rigid body. A thin layer made of a light, stiff fictitious material is inserted between the steel cylinder and the granular medium in order to ensure a contact length that does not depend on the geometry of the massive object. The contact length $l$ is defined such that the contact filling ratio of the $2 \mathrm{D}$ unit cell matches that of the $3 \mathrm{D}$ unit cell: $l / l_{\mathrm{UC}}=S / l_{\mathrm{UC}}^{2}$, with $S=l_{\mathrm{UC}}^{2} \pi / 4$, which gives $l \approx 0.94 \mathrm{~cm}$. The height $D^{(2 \mathrm{D})}$ is taken such that the consolidation magnitude $z_{0}$ is kept constant: $D^{(2 \mathrm{D})}=l$ for the heavy case and $D^{(2 \mathrm{D})}=l / 4 \approx 0.24 \mathrm{~cm}$ for the light case. Finally, when taken into account, the roots are modeled as a single steel central pillar, with a height of $4 \mathrm{~cm}$ and a width $w_{\text {root }}$ such that $w_{\text {root }} / l$ is equal to the ratio of the cross section of all wires to the contact area: we take $w_{\text {root }} / l=1 / 25$.

From this 2D model, dispersion analyses are run by sweeping the Floquet wave number $k$ in the longitudinal direction in the range $k \in\left(0, \pi / l_{\mathrm{UC}}\right)$ and obtaining the eigenfrequencies at each step. A typical result for a heavy object is represented in Fig. 4. One can clearly see in Fig. 4(a) that the first two modes hybridize and asymptotically tend to flat frequency curves, while higher-order modes are less affected. As highlighted by insets (b1) and (b2), these hybridizations are associated with resonances involving a rigid body motion of the massive solid: mode no. 1 is linked to the vertical motion while mode no. 2 is linked to the longitudinal one. Parametric studies show that these asymptotic values can be tuned independently by changing the mass and angular momentum of the solid.

\section{Transmission analysis through a single line of scatterers}

The dispersion analysis is complemented with a study on the modal insulation capabilities of a single line of periodically spaced scatterers, as a function of frequency. To this purpose, a finite-element model is built with the following characteristics [see Fig. 3(b)]. An object is placed at the middle of a region of interest, which is large enough to include a dozen wavelengths of propagation on each side and is surrounded with perfectly matched layers (PML). The massive object and its induced consolidation have the same characteristics as in the dispersion analysis. Waves are generated by a time-harmonic line source $e^{i \omega t} \delta\left(x-x_{S}\right) \delta\left(z-z_{S}\right) \mathbf{F}$ with a force vector $\mathbf{F}=$ $\left(F_{x}, 0, F_{z}\right)^{T}$ and depth location $z_{S}$ chosen to selectively excite one mode. This solution is not as elegant as inserting the exact modal shape as excitation distribution, but is realistic to implement experimentally and proves to be effective enough for the first three modes. Based on an elementary analysis of the location of the nodes of the 

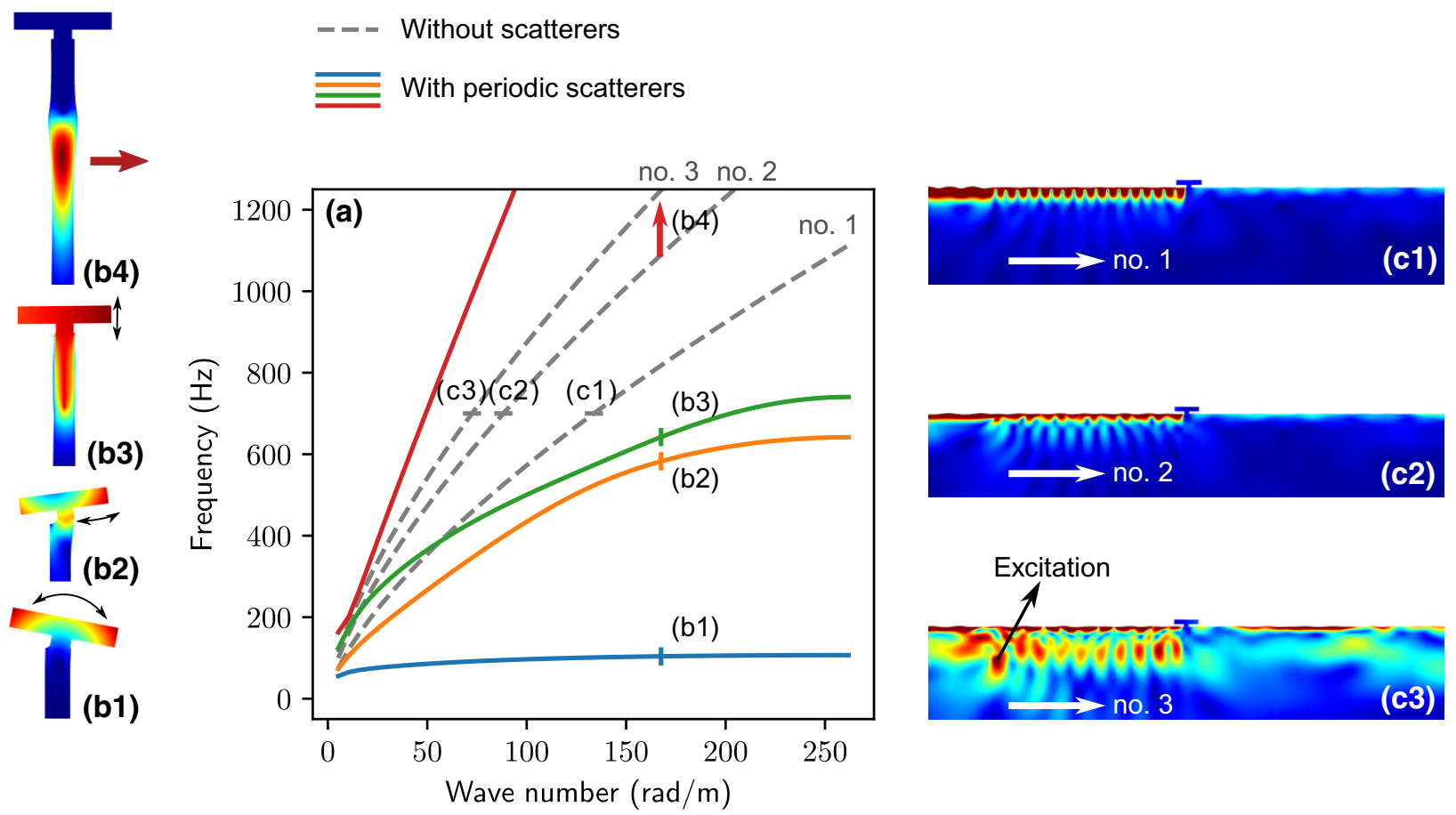

FIG. 4. Numerical study on a 2D model of a heavy scatterer: (b1)-(b4) first four mode shapes for a periodic arrangement of scatterers displayed at a quarter-wavelength scale $(d / \lambda=4)$ and (a) dispersion curves (solid) compared to the reference medium (dashed), showing a high hybridization of modes no. 1 and no. 2 with longitudinal and vertical rigid-body-like resonances of the massive object. (b4) shows that at higher frequencies the medium behaves as a waveguide with clamped surface. (c1) -(c3) analysis of transmission through a single scatterer for (quasi) pure modal incidence, at $700 \mathrm{~Hz}$.

lowest-order mode shapes at $700 \mathrm{~Hz}$, we identify that mode no. 1 can be reasonably selectively excited by taking $\left\{z_{S}, \mathbf{F}\right\}_{\text {no. } 1}(700 \mathrm{~Hz})=\left\{1.7 \mathrm{~cm},(0,0,1)^{T}\right\}$, mode no. 2 with $\left\{z_{S}, \mathbf{F}\right\}_{\text {no. } 2}(700 \mathrm{~Hz})=\left\{1.2 \mathrm{~cm},(1,0,0)^{T}\right\}$ and mode no. 3 with $\left\{z_{S}, \mathbf{F}\right\}_{\text {no. } 3}(700 \mathrm{~Hz})=\left\{7.1 \mathrm{~cm},(0,0,1)^{T}\right\}$. We use the spatial scaling given in Eq. (2) to extrapolate these recipes at other frequencies: $z_{S}(f)=z_{S}(700 \mathrm{~Hz}) \times r$ with $r=(700 \mathrm{~Hz} / f)^{1 /(1-\alpha)}$ the scaling factor. All dimensions relative to the propagation window are also multiplied by $r$ to follow the frequency scaling, so that the number of nodes remains under control over a wide frequency range (about $2 \times 10^{5}$ degrees of freedom) and calculations can be run routinely on a personal computer at moderate costs. A $2 \mathrm{D}$ model is validated against a $3 \mathrm{D}$ model that relies on periodic conditions in the transverse direction (so that only one slice is meshed) and then exploited for parametric studies. As represented in Figs. 4(c1)-4(c3), the outcomes confirm that the hybridization of modes no. 1 and no. 2 observed with the dispersion analysis indeed result in a low transmission, while modes no. 3 and higher transmit most of their energy without scattering significantly. These transmission ratios are measured by averaging the amplitude of the vertical motion at the surface over a window long of several wavelengths and located behind the line of scatterers (see Fig. 3), and by normalizing by the amplitude obtained in the absence of scatterers. Variations of this model are also implemented to analyze separately the roles played by the different constituents: the roots may be included or not, the object may be replaced by a clamping boundary condition acting over a pillar that may be considered consolidated or not. Results are presented in the next section in a comparison with experiments.

\section{EXPERIMENTAL RESULTS}

Experiments are conducted for the two sets of scatterers described above, labeled "light" and "heavy" further on (their mass ratio is of 1 to 4). A typical result for a line of heavy objects is presented in Fig. 5. One can clearly recognize qualitatively the behavior expected from the numerical analysis, namely that fast (high-order) modes are well transmitted while slow (the first two) modes are efficiently stopped. Down-conversion from fast to slow is visible, although because fast modes are essentially localized in a deep channel this interaction is actually largely inefficient from an energetic point of view. Concerning the significant transmission occurring around $t=14 \mathrm{~ms}$, a time-frequency analysis shows that it is a too early event to be a no. $2 \rightarrow$ no. 2 transmission, and is indeed mostly a no. $3 \rightarrow$ no. 2 down-conversion. More quantitative insight can be gained with a deeper data processing as performed below (see Appendix B for more details). 


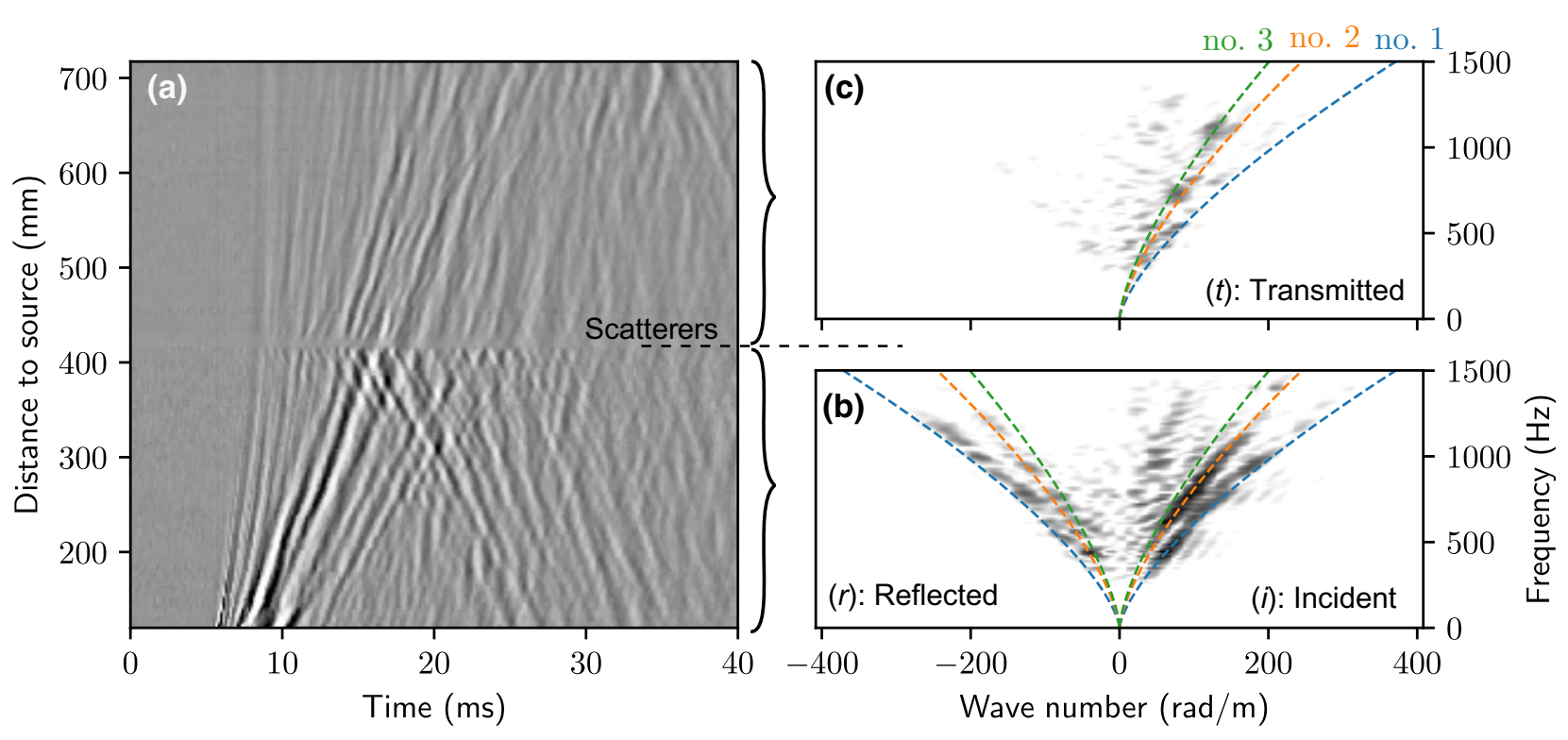

FIG. 5. Vertical velocity of a field interacting with one line of "heavy" scatterers located at $420 \mathrm{~mm}$ from the source: (a) space-time signals, (b),(c) experimental dispersion curves obtained from the first (120 to $420 \mathrm{~mm}$ ) and second ( 420 to $720 \mathrm{~mm}$ ) halves of the data, together with theoretical curves (dashed) of the first three modes.

\section{A. Comparison with models}

The comparison of the transmission ratios extracted from the experiments with those predicted by the 2D numerical models is presented in Fig. 6. The good agreement proves the relevance of the simplification assumptions made to account for the role of additional consolidation and roots. The achieved effect is a low-pass filter on modes no. 1 and no. 2. The ratio of contact area to wavelength is a key parameter, but significant blocking is obtained at a moderately subwavelength scale: the contact area diameter is $1.2 \mathrm{~cm}$ while $\lambda_{1}(700 \mathrm{~Hz})=4.7 \mathrm{~cm}$ and $\lambda_{2}(1000 \mathrm{~Hz})=4.25 \mathrm{~cm}$, so that transmission ratios about $25 \%(-12 \mathrm{~dB})$ are reached at $\lambda / 4-\lambda / 3$. Though broadband, the effect arises from a resonant origin as highlighted by the model without roots. Above resonance, which for the heavy object occurs at $230 \mathrm{~Hz}$ (longitudinal) and $260 \mathrm{~Hz}$ (vertical), the boundary condition schematically changes from free to clamped, which, together with the additional consolidation, results in a mismatch of mode shapes and in an accordingly high modal impedance barrier whenever the incident wave is localized near the surface. Of course such an idealized massive object without roots would not stand in practice without sinking. The solution retained here to add roots has the consequence to damp the resonance to such a low-quality factor that its peak is no longer noticeable, but preserves the broadband high frequency effect. Nevertheless, recovering a higher quality factor - by improving the device with, for instance, a spring between the mass and the roots - is attractive as transmission of mode no. 1 could be kept at low levels from much deeper subwavelength scales [here $\lambda_{1}(260 \mathrm{~Hz}) \approx 20 \mathrm{~cm}$, so the scale of the interaction is about $D / \lambda_{1} \approx \lambda_{1} / 15$ while the numerical model reaches $-11 \mathrm{~dB}$ transmission]. The extra effect brought by a higher quality factor is less obvious for mode no. 2. We believe that the principal reason is that the configuration involves a too extremely deep subwavelength scale [here $\lambda_{2}(230 \mathrm{~Hz}) \approx 35 \mathrm{~cm}$, so $\left.D / \lambda_{2} \approx \lambda_{1} / 30\right]$.

\section{CONCLUSIONS}

A metabarrier is introduced, featuring a low-pass filter like transmission properties at subwavelength scales for the two fundamental modes guided at the surface of an unconsolidated granular half-space. It relies on a rigid, massive object standing at the surface of the soft, locally compressed substrate, the coupling of both resulting in a mass-spring system on longitudinal and vertical components with low-quality factors. The practical solution adopted to build such scatterers and make them stand on the fragile surface involves roots, which do not hinder the transmitting to insulating transition, but whose adverse effect is to lower the quality factors to such a point that no noticeable deeply subwavelength effect occurs at the resonance frequencies. Based on numerical models showing good agreement with an experimental realization, two essential ingredients are identified to jointly contribute to the broadband insulation effect. The first element is the effective clamping of the surface at high frequencies, which is in turn a consequence of the resonant mass-soil coupling. The second element is the consolidation of the soil below the barrier, which is a consequence of the added pressure at the surface. This extra consolidation induces 


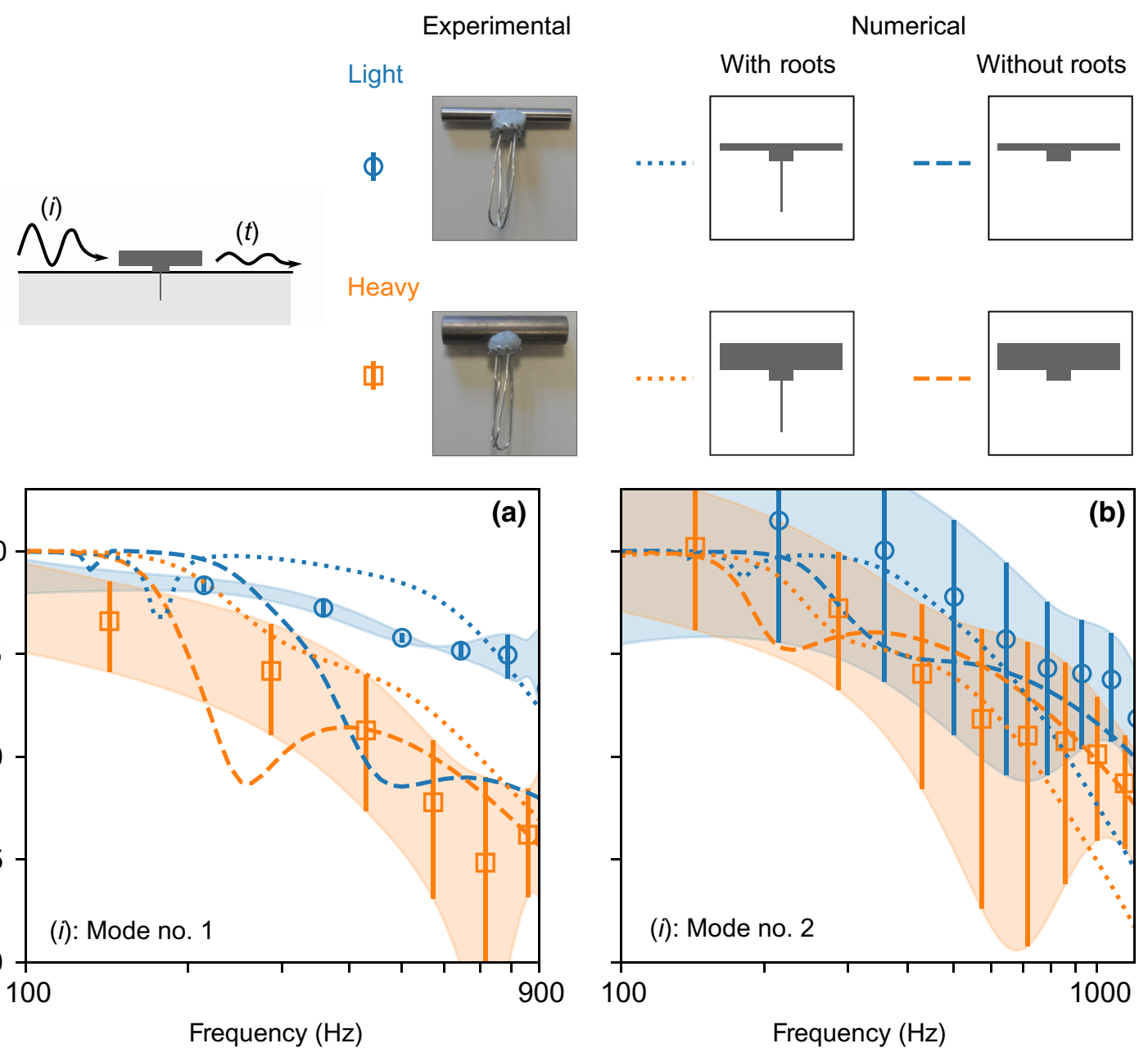

FIG. 6. Transmission ratio of vertical motion at the surface through one line of light (blue) and heavy (orange) scatterers, for incident modes (a) no. 1 and (b) no. 2: (symbols) experimental values against numerical models (dotted) with or (dashed) without account of roots.

a change of scale in the depth profiles below the barrier and ensures that the clamping of the surface indeed results in a low motion over the entire energy distribution of incoming, weakly penetrating modes, which then undergo a high impedance mismatch. The role of each resonance is highlighted: mode no. 1 hybridizes principally with a vertical clamping, as already reported, while mode no. 2 requires a longitudinal one to be efficiently affected. As suggested by a model, an attractive improvement to this study - although at the cost of the extreme simplicity of the scatterers - would be to add springs between the mass and the roots so as to recover strong hybridization around the resonant frequencies and achieve low transmission properties starting from deeper subwavelength scales and with lighter masses.

These results contribute to a better understanding of the control of seismic waves by metamaterials placed on the surface of elastic layers. The concept of adding a rigid mass with an appropriate design on the granular elastic system is shown to be suitable for the realization of an efficient seismic barrier, and adds to the previously studied strategies. It is therefore possible to imagine the design of relatively simple seismic metamaterials, capable of operating advanced effects on the propagation of complex subsurface modes, such as trapping, focusing, total shielding, refraction, etc.

\section{ACKNOWLEDGMENTS}

We acknowledge H. Méziere, J. Marudai and E. Egon for manufacturing the mechanical parts and E. Brasseur for his assistance in setting up the experiment.

\section{APPENDIX A: EXPERIMENTAL SET-UP}

The experimental setup shown in Fig. 1 derives from the ones of Refs. [14-18,20]. The dimensions of the sand box are $100 \times 55 \times 30 \mathrm{~cm}$, with a filling height of $25 \mathrm{~cm}$. The granular medium is composed of calibrated silica beads 
of diameter $180 \mu \mathrm{m}$ and has a packing density of $\rho=$ $1560 \mathrm{~kg} / \mathrm{m}^{3}$. Beads are frequently stirred between experiments to limit accumulation of consolidation with time, and roughly leveled to ensure a reasonably flat surface. For the elastic wave source, a B\&K V201 shaker driven by an Agilent 33120A function generator connected to a B\&K LDS LPA100 power amplifier, excites a screwed rod embedded into the granular medium with a vertical motion of a few millimeters' amplitude. The chosen excitation signal is a Ricker pulse, centered in frequency at $2 \mathrm{kHz}$. The source is located at a distance of $1 \mathrm{~cm}$ to a wall of the box so as to avoid impulse echos and to produce a radiation pattern which is close to cylindrical for the involved wavelengths (around $4 \mathrm{~cm}$ for the shortest one). We measure the vertical particle velocity at the surface with a Polytech laser Doppler velocimetry head. The head is moved with a Newport XPS-RL Motion Controller along an axis within a range of $60 \mathrm{~cm}$ starting from a distance to the source of about $12 \mathrm{~cm}$. The vertical velocity is measured at 230 steps $(\Delta x \approx 2.6 \mathrm{~mm})$ along this axis and digitized with a Zurich MFLI card, each $130 \mathrm{~ms}$ long signal being averaged 20 times and sampled at a rate of $29.3 \mathrm{kHz}$.

The metabarrier is made of steel rods (density $\rho_{S} \approx$ $7800 \mathrm{~kg} / \mathrm{m}^{3}$, length $5 \mathrm{~cm}$, diameter $1 / 4 \mathrm{in}$. for light and $1 / 2$ in. for heavy objects, i.e., masses of, respectively, 11 and $45 \mathrm{~g}$ ) arranged in a row, which splits the measurement line in two parts of $30 \mathrm{~cm}$ each. The rods stand on the sand on a controlled area of $1.2 \mathrm{~cm}$ diameter by means of an attached washer, itself connected to thin roots made of a metallic wire to avoid sinking. The rods are lain horizontally on the washers to maximize the inertial momentum. To prevent newly deposited scatterers from strongly disturbing the fragile medium and making previously located ones sink, this preparation relies on first protecting the surface with two sheets of paper, inserting the objects between both, and finally carefully removing the sheets.

\section{APPENDIX B: DATA PROCESSING}

The objective of the data processing described here is to extract transmission ratios of pure modal incidence from the recorded signals. Each $x-t$ map of signals is split along the abscissa of the interface $x_{S}$ into a map of incident and reflected waves for $x<x_{S}$, and a map of transmitted waves for $x>x_{S}$, which are then processed independently. We first clean outliers with a median filter on the $x-t$ maps with a patch size $3 \times 3$. We then proceed to a mode selection in the 2D-Fourier-transformed domain by windowing the $\omega-k$ plane around the dispersion relation meant to be isolated. Finally, we select the wave train of appropriate arrival by time windowing the phasecompensated signals. This way, maps of no. $1 \rightarrow$ no. 1 , no. $1 \rightarrow$ no. 2 , no. $2 \rightarrow$ no. 1 and no. $2 \rightarrow$ no. 2 transmissions are obtained and combined into maps of no. $1 \rightarrow$ (no. $1+$ no. 2 ) and no. $2 \rightarrow$ (no. $1+$ no. 2 ) transmissions.
From these latter maps, we measure the incident and transmitted amplitudes by averaging the power spectrum over spatial windows close to either sides of the interface. As this procedure is rather sensitive to the dispersion relation used for Fourier windowing and phase compensation, $\omega_{1}(k)$ and $\omega_{2}(k)$ are carefully adjusted for each new set of acquisitions. This is especially true for mode no. 2, which is not easily separated from mode no. 3 . In order to account for biases due to this processing, to dissipation, and to geometrical attenuation of the beams, the same procedure is applied to reference maps of signals measured without scatterers, and the obtained transmission ratios are used as normalization factors. Finally, confidence intervals and average values are estimated from repeated measurements.

[1] S. Brûlé, S. Enoch, and S. Guenneau, Emergence of seismic metamaterials: Current state and future perspectives, Phys. Lett. Sect. A: Gen. At., Solid State Phys. 384, 126034 (2020).

[2] S. Brûlé, E. Javelaud, S. Enoch, and S. Guenneau, Experiments on Seismic Metamaterials: Molding Surface Waves, Phys. Rev. Lett. 112, 133901 (2014).

[3] A. Colombi, P. Roux, S. Guenneau, P. Gueguen, and R. V. Craster, Forests as a natural seismic metamaterial: Rayleigh wave bandgaps induced by local resonances, Sci. Rep. 6, 1 (2016).

[4] P. Roux, D. Bindi, T. Boxberger, A. Colombi, F. Cotton, I. Douste-Bacque, S. Garambois, P. Gueguen, G. Hillers, D. Hollis, T. Lecocq, and I. Pondaven, Toward seismic metamaterials: The METAFORET project, Seismol. Res. Lett. 89, 582 (2018).

[5] A. Palermo, S. Krödel, K. H. Matlack, R. Zaccherini, V. K. Dertimanis, E. N. Chatzi, A. Marzani, and C. Daraio, Hybridization of Guided Surface Acoustic Modes in Unconsolidated Granular Media by a Resonant Metasurface, Phys. Rev. Appl. 9, 054026 (2018).

[6] Y. Chen, F. Qian, F. Scarpa, L. Zuo, and X. Zhuang, Harnessing multi-layered soil to design seismic metamaterials with ultralow frequency band gaps, Mater. Des. 175, 107813 (2019).

[7] R. Zaccherini, A. Colombi, A. Palermo, V. K. Dertimanis, A. Marzani, H. R. Thomsen, B. Stojadinovic, and E. N. Chatzi, Locally Resonant Metasurfaces for Shear Waves in Granular Media, Phys. Rev. Appl. 13, 034055 (2020).

[8] A. Colombi, D. Colquitt, P. Roux, S. Guenneau, and R. V. Craster, A seismic metamaterial: The resonant metawedge, Sci. Rep. 6, 1 (2016).

[9] M. Miniaci, A. Krushynska, F. Bosia, and N. M. Pugno, Large scale mechanical metamaterials as seismic shields, New J. Phys. 18, 083041 (2016).

[10] Y. Achaoui, B. Ungureanu, S. Enoch, S. Brûlé, and S. Guenneau, Seismic waves damping with arrays of inertial resonators, Extreme Mech. Lett. 8, 30 (2016), nanomechanics: Bridging Spatial and Temporal Scales.

[11] Y. Achaoui, T. Antonakakis, S. Brûlé, R. V. Craster, S. Enoch, and S. Guenneau, Clamped seismic metamaterials: 
Ultra-low frequency stop bands, New J. Phys. 19, 063022 (2017).

[12] A. Maurel, J.-J. Marigo, K. Pham, and S. Guenneau, Conversion of love waves in a forest of trees, Phys. Rev. B 98, 134311 (2018).

[13] K. Pham, S. Maurel, A. Félix, and S. Guenneau, Hybridized love waves in a guiding layer supporting an array of plates with decorative endings, Materials 13, 1632 (2020).

[14] X. Jacob, V. Aleshin, V. Tournat, P. Leclaire, W. Lauriks, and V. E. Gusev, Acoustic Probing of the Jamming Transition in an Unconsolidated Granular Medium, Phys. Rev. Lett. 100, 158003 (2008).

[15] L. Bodet, X. Jacob, V. Tournat, R. Mourgues, and V. Gusev, Elasticity profile of an unconsolidated granular medium inferred from guided waves: Toward acoustic monitoring of analogue models, Tectonophysics 496, 99 (2010).

[16] L. Bodet, A. Dhemaied, R. Martin, R. Mourgues, F. Rejiba, and V. Tournat, Small-scale physical modeling of seismicwave propagation using unconsolidated granular media, Geophysics 79, T323 (2014).

[17] P. Bergamo, L. Bodet, L. V. Socco, R. Mourgues, and V. Tournat, Physical modelling of a surface-wave survey over a laterally varying granular medium with property contrasts and velocity gradients, Geophys. J. Int. 197, 233 (2014).

[18] S. Pasquet, L. Bodet, P. Bergamo, R. Guérin, R. Martin, R. Mourgues, and V. Tournat, Small-scale seismic monitoring of varying water levels in granular media, Vadose Zone J. 15, vzj2015.11.0142 (2016).
[19] V. Gusev, V. Aleshin, and V. Tournat, Acoustic Waves in an Elastic Channel Near the Free Surface of Granular Media, Phys. Rev. Lett. 96, 214301 (2006).

[20] V. Aleshin, V. Gusev, and V. Tournat, Acoustic modes propagating along the free surface of granular media, J. Acoust. Soc. Am. 121, 2600 (2007).

[21] V. Tournat and V. Gusev, Acoustics of unconsolidated "model" granular media: An overview of recent results and several open problems, Acta Acust. United Acust. 96, 208 (2010).

[22] L. Bonneau, B. Andreotti, and E. Clément, Evidence of Rayleigh-Hertz Surface Waves and Shear Stiffness Anomaly in Granular Media, Phys. Rev. Lett. 101, 118001 (2008).

[23] G. Gladwell, The calculation of mechanical impedances relating to an indenter vibrating on the surface of a semi-infinite elastic body, J. Sound. Vib. 8, 215 (1968).

[24] K. L. Johnson, Contact Mechanics (Cambridge University Press, Cambridge, 1987).

[25] N. Boechler, J. K. Eliason, A. Kumar, A. A. Maznev, K. A. Nelson, and N. Fang, Interaction of a Contact Resonance of Microspheres with Surface Acoustic Waves, Phys. Rev. Lett. 111, 036103 (2013).

[26] M. Hiraiwa, M. Abi Ghanem, S. P. Wallen, A. Khanolkar, A. A. Maznev, and N. Boechler, Complex Contact-Based Dynamics of Microsphere Monolayers Revealed by Resonant Attenuation of Surface Acoustic Waves, Phys. Rev. Lett. 116, 198001 (2016). 